\title{
Idiopathic Bradycardia in Unicuspid Unicommissural Aortic Valve Complicated with Infective Endocarditis and Aortic Root Abscess: A Case Report
}

\author{
Mohammed Abd Al Jawad, ${ }^{1}$ Khaled Refaat, ${ }^{1}$ Mohammed Attiya, ${ }^{1}$ Nada Abdelhakim, ${ }^{2}$ Samar Abdeltawab ${ }^{2}$ \\ ${ }^{1}$ Department of Cardiothoracic Surgery, Ain Shams University, Cairo, Egypt; \\ ${ }^{2}$ Department of Anesthesia and ICU, Ain Shams University, Cairo, Egypt
}

\section{ABSTRACT}

Unicuspid aortic valves are among the rarest congenital malformations. They are classified as either acommissural or unicommissural, with the unicommissural being presented in early adulthood. Unicuspid valves share many similarities with bicuspid valves, namely increased rates of valve degeneration and calcification, making them prone to secondary aortic stenosis, regurgitation, or both. Among other similarities are the increased risk of aortic root dilatation, dissection, and left ventricular dilatation. We report our case of a 23 -year-old male with unicuspid unicommissural aortic valve with aortic root and left ventricular dilatation. He successfully underwent Wheat procedure.

\section{INTRODUCTION}

Unicuspid aortic valve is an extremely rare malformation with a prevalence as low as $0.02 \%$ in the adult population. They usually come in two varieties: the more aggressive acommissural type, which is usually presented in early childhood, and the less virulent unicommissural type [Mookadam 2010; Mookadam 2010].

In a country with rheumatic fever as a major player in multi-valvular affection, it is almost always to associate isolated aortic valve affection without mitral involvement to a congenital anomaly [Roberts 2005].

\section{CASE REPORT}

A 23-year-old male patient was presented to his cardiologist with progressive exertional dyspnea. On auscultation, he had a combination of systolic and diastolic murmurs over the left parasternal region. Transthoracic echocardiographic examination revealed a unicuspid sclerotic aortic valve with

Received February 8, 2021; accepted March 9, 2021.

Correspondence: Dr. Mohammed Abd AL Jawad, MD, PbD, Associate Professor of Cardiothoracic Surgery, Department of Cardiothoracic Surgery, Ain Shams University, Abbaseya Square, Cairo, Egypt, Telephone +201111219461 (e-mail: mohammed_abdaljawad@med.asu.edu.eg) severe AS (Vmax $4.1 \mathrm{~m} / \mathrm{s}$, peak pressure gradient of $66 \mathrm{mmHg}$, mean pressure gradient of $42 \mathrm{mmHg}, \mathrm{DVI}=0.22$, aortic valve area AVA by continuity $1.5 \mathrm{~cm}^{2}$ ), severe eccentric AR (end diastolic reversal at descending aorta $=26 \mathrm{~m} / \mathrm{sec}$ ) dilated $\mathrm{LV}$ internal dimensions and mildly dilated aortic root $4.1 \mathrm{~cm}$. His electrocardiogram revealed periods of unexplained sinus bradycardia. The patient had no clinical picture or family history of connective tissue disease or infective endocarditis. His laboratory findings were unremarkable (weight $65 \mathrm{Kg}$, height $180 \mathrm{~cm}$, BSA $1.83 \mathrm{~m}^{2}$ ).

Multi slice CT revealed a unicuspid calcified aortic valve with calcium score 3591, left-sided bovine aortic arch with two branches; the first common trunk gives the brachiocephalic trunk and left common carotid artery, while the second is the left subclavian artery. Aortic diameter at level of sinus of Valsalva $4.4 \times 3.4 \mathrm{~cm}$, at level of sinotubular junction 3.1 x 2.9; and $2.3 \times 2.3 \mathrm{~cm}$ just before the brachiocephalic trunk. (Figure 1) (Supplementary Figures 1-4)

Intraoperative transesophageal echocardiography confirmed the diagnosis of a unicuspid unicommissural aortic valve, and the presence of a heavily calcified mass and a small freely mobile vegetation at the cusp free edge was noticed. (Figure 2) (Video 1)

During the operation, the patient had periods of unexplained sinus bradycardia. Following pericardiotomy, there was a saccular dilatation of the ascending aorta above the level of sinutubular junction until just before take-off of the brachiocephalic trunk. After cardioplegic arrest of the heart, complete transection of the aorta with inspection of the valve again confirmed the diagnosis of unicuspid unicommissural

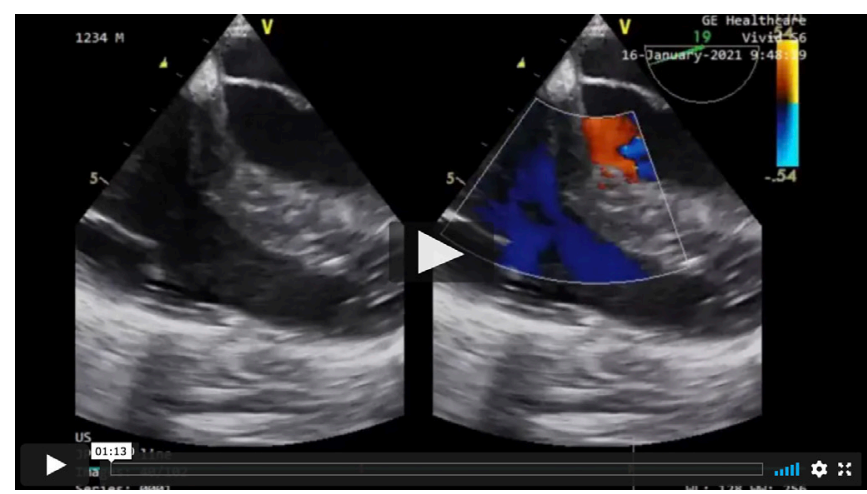

Video 1. Intraoperative TEE 

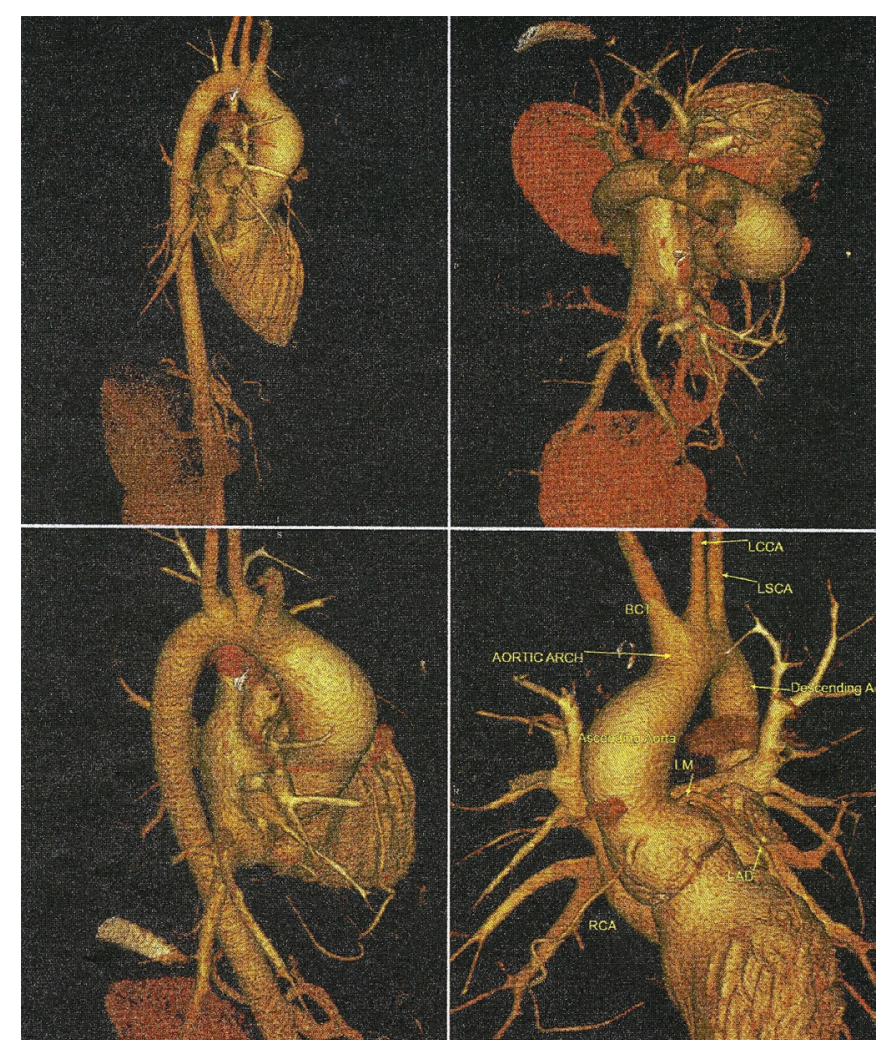

Figure 1. MSCT 3D reconstruction showing dilatation of ascending aorta above level of STJ. heavily calcific aortic valve, eccentric slit opening between left and non-coronary cusps with one commissural attachment to the wall. (Figure 3)

During excision of the valve, a tiny forming abscess cavity was noted at the right and non-coronary cusps partially encroaching on the membranous septum (Figure 3D), which was drained and obliterated by double-armed Teflon pledgeted sutures. This was followed by mechanical aortic valve replacement by $21 \mathrm{St}$ Jude Medical (SJM) Regent (St Jude Medical, St Paul, Minnesota, USA) and was completed by a $30 \mathrm{~mm}$ Hemashield Platinum Woven Double Velour Vascular interposition graft (Getinge, La Ciotat, France) to replace the ascending aorta from the level of STJ until $1 \mathrm{~cm}$ before the origin of the brachiocephalic trunk.

The cytochemical analysis of the drained fluid confirmed the purulent nature rather than liquified calcium. The excised tissue and postoperative cultures returned no growth under aerobic and anerobic conditions. The patient showed an excellent ICU and ward recovery and was discharged home after one week. The patient completed three uneventful follow-up visits; in each visit an electrocardiogram and a 48 hours Holter ECG were done showing no rhythm defects.

\section{DISCUSSION}

The main distinguishing feature of our report is the presence of repeated episodes of preoperative sinus bradycardia, which is not a common association in the already very rare pathology of unicuspid unicommissural aortic valve.
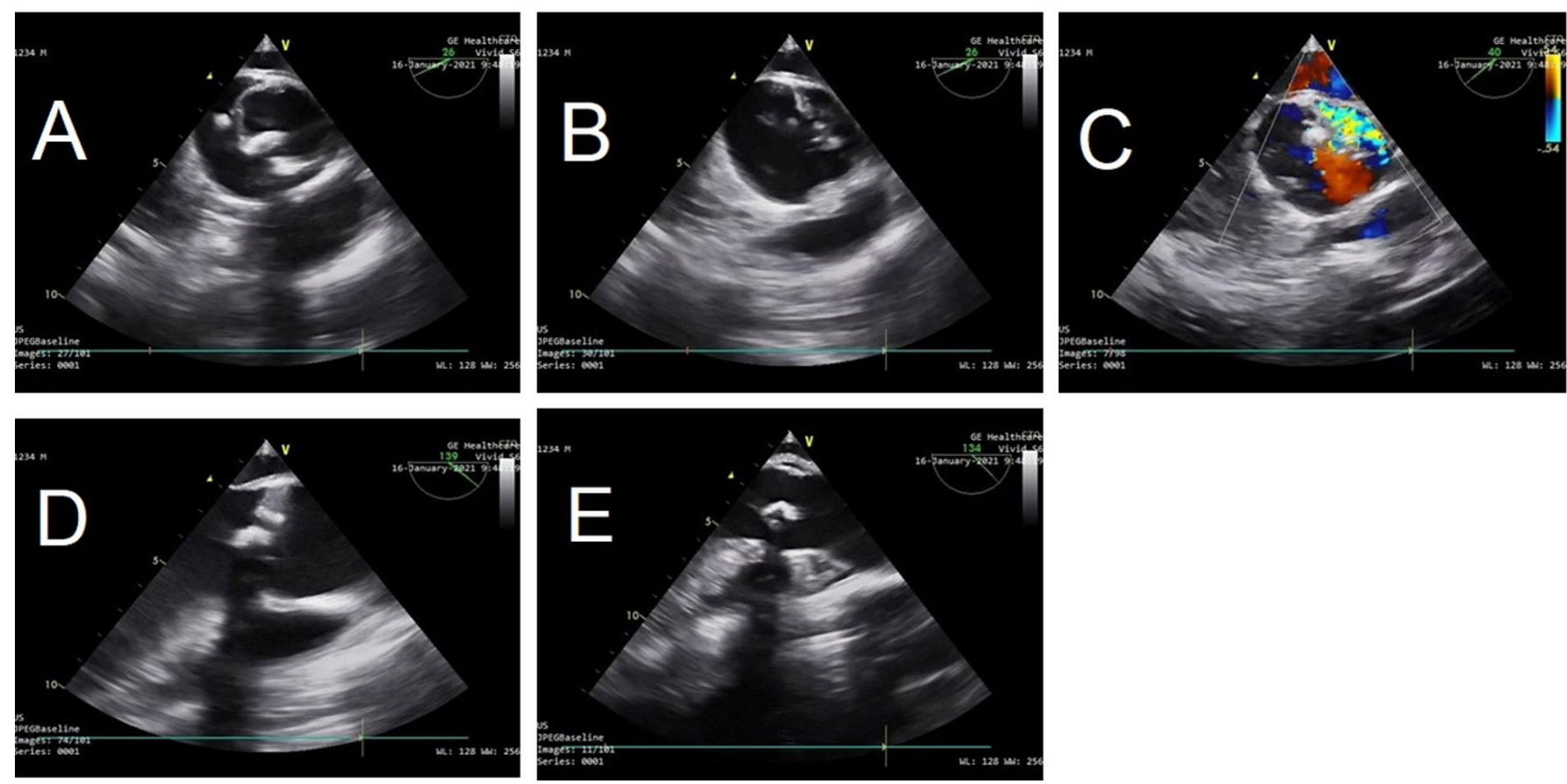

Figure 2. Preoperative TEE. A) Aortic valve shot axis opening in systole; B) Aortic valve short axis showing valve closure in diastole showing large defect; C) Aortic valve short axis with color flow doppler showing flow of regurgitation during valve closure; D) Aortic valve long axis showing valve closed in diastole with calcified masses on free edge; E) Aortic valve long axis showing valve closed in diastole with calcified masses on free edge. 
Repairing the valve was not feasible due to the presence of heavy calcification. The surgical decision was to do a mechanical aortic valve replacement due to the patient's young age and an acceptable level of education and comprehension of his medical condition and requirement of life long anti-coagulation therapy.

On the other hand, the aortic root dilatation was a matter of debate. Being of $41 \mathrm{~mm}$ at its widest diameter and not reaching the $45 \mathrm{~mm}$ mark of ESC/EACTS guidelines [2014 ESC Guidelines 2015], absence of relevant family history, connective tissue disease and diameters of adjacent aortic segments, the verdict was to replace the apparently dilated segment by an interposition graft as the patient was refusing a major Bentall procedure.

We have encountered preoperative sinus bradycardia, to which there was no apparent explanation, taking into consideration no prior diagnosis of infective endocarditis. However, during valve excision, the forming abscess cavity was in very close proximity to the conduction system. We believe it might serve as an irritant to it, causing bradycardia. There was no need for a postoperative pacemaker, and the patient did not develop any new episode of bradycardia or heart block like before. To our knowledge, only postoperative heart block was reported due to the technical and anatomical features of aortic valve replacement itself, however this was not the explanation to our findings [Singh 2015; Ravi 2019].

There are a few similar reports of unicuspid malformation and infective endocarditis [Chalwade 2019; Yuzawa-Tsukada
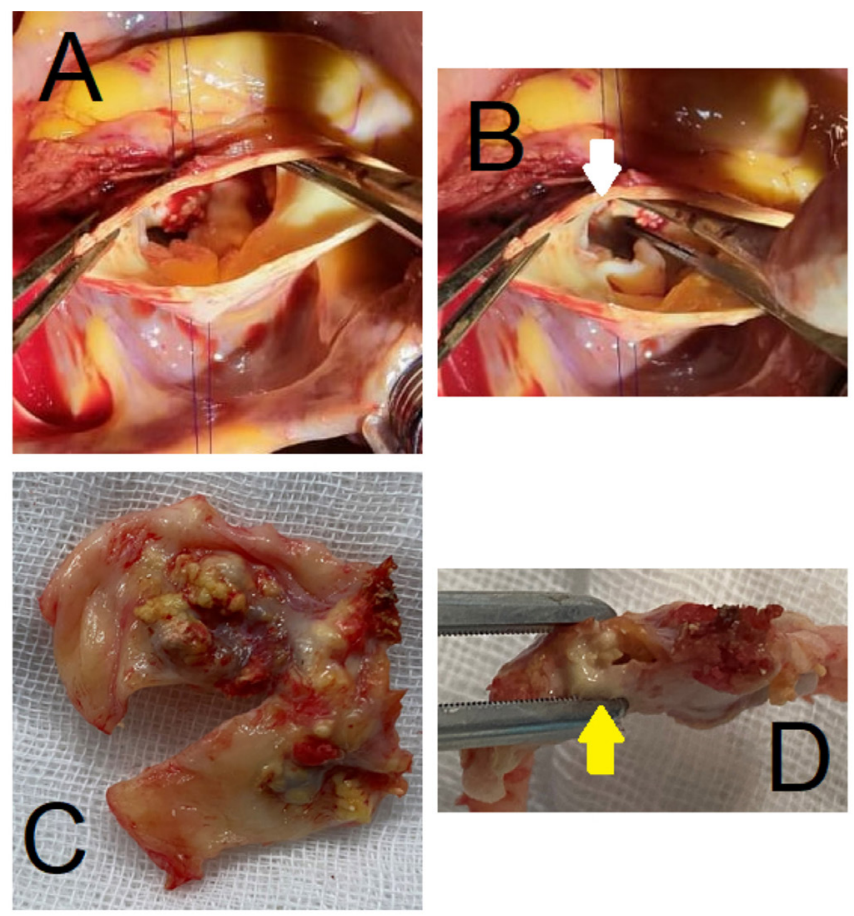

Figure 3. Intraoperative images of aortic valve. A) Surgeon's perspective of the transected aorta and valve; B) Yellow arrow denoting valve opening; C) Totally excised valve (one piece) anatomically configured as surgeon's point of view; D) White arrow is the part of the abscess cavity at the base of the valve with pus formed inside.
2019]. In these reports, a well-established preoperative diagnosis of infective endocarditis exists, unlike our report of a "possible" diagnosis of infective endocarditis. Rather than "definite or rejected," we lacked the definite microorganism culture, yet we had the gross pathological intracardiac abscess during surgery, along with valve degeneration and

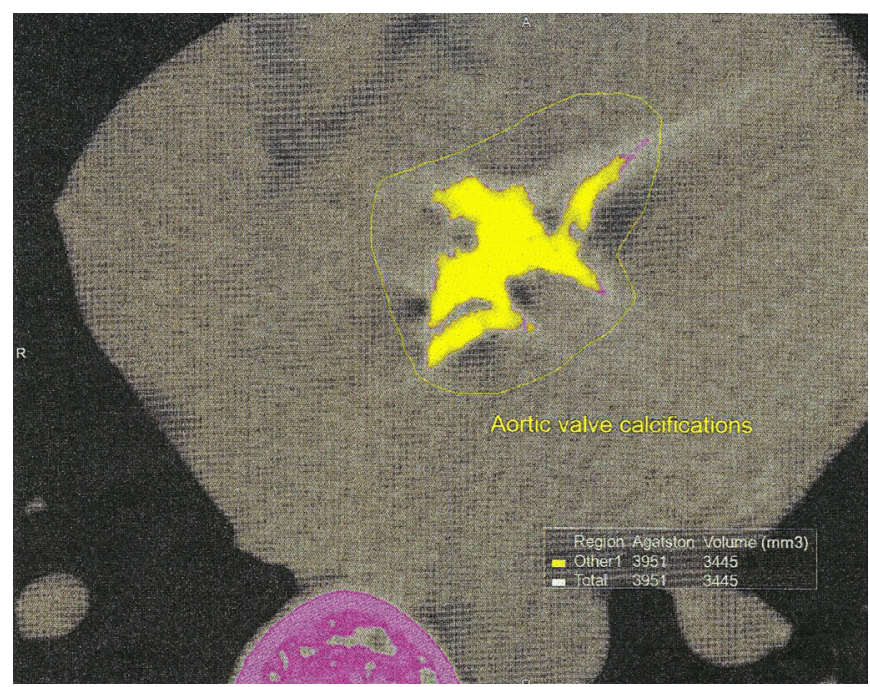

Supplementary Figure 1. MSCT showing aortic valve calcifications.

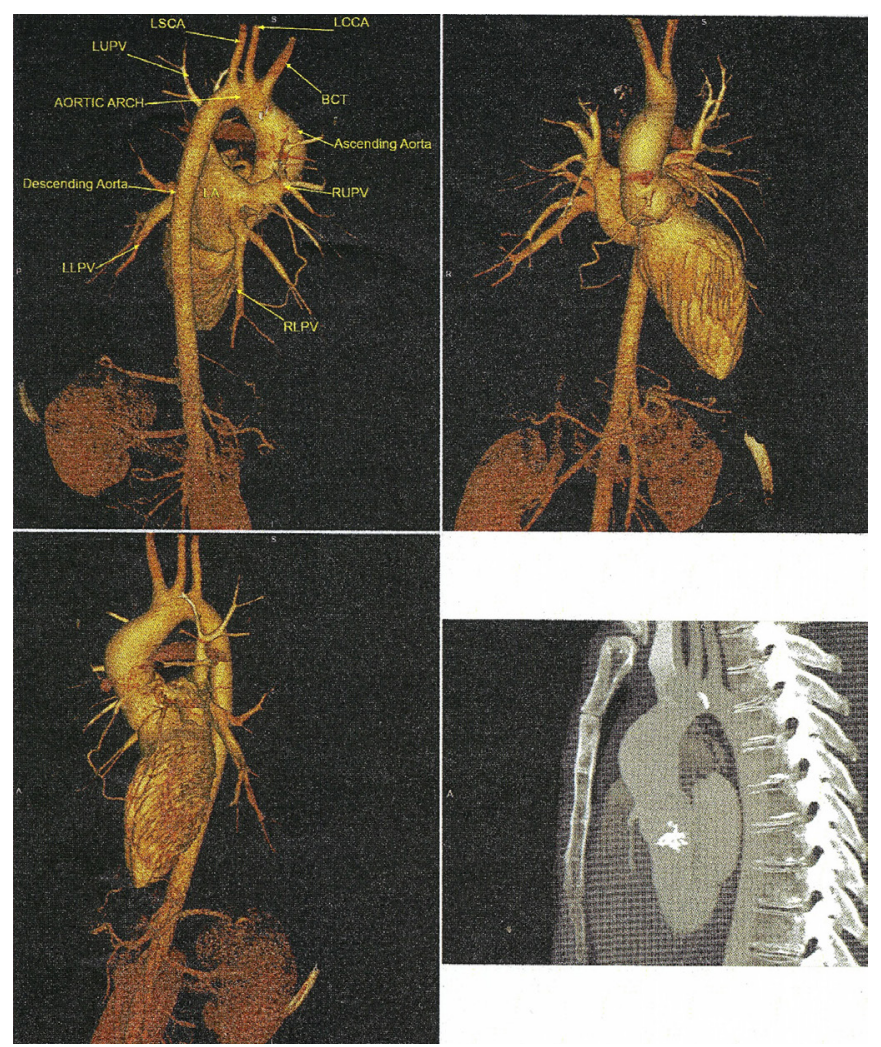

Supplementary Figure 2. MSCT 2D/3D showing bovine aortic arch. 

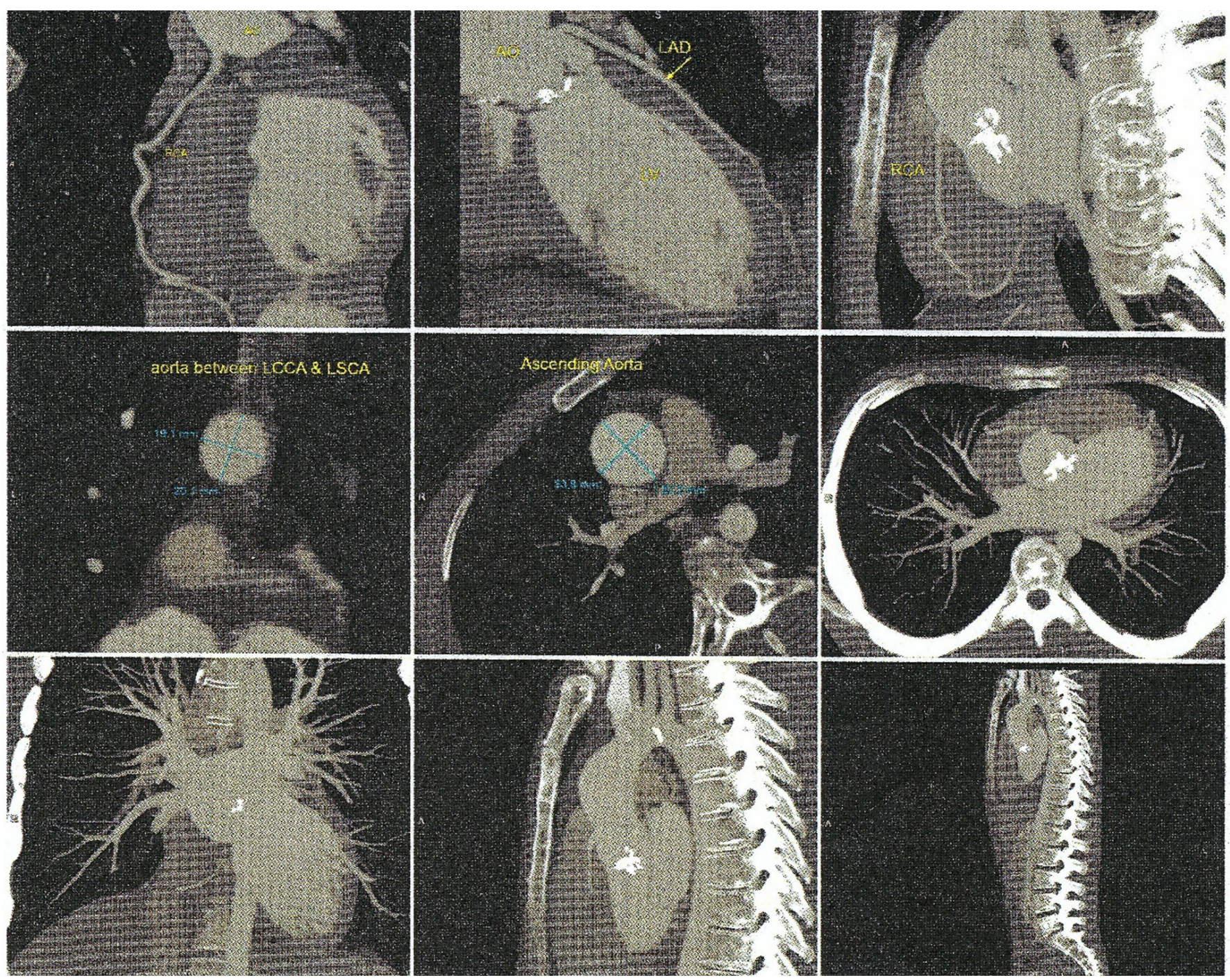

Supplementary Figure 3. MSCT showing aortic measurements at different levels.

calcification. We also had the absence of alternate diagnosis to explain the findings. In our case, we considered ourselves lucky, since we encountered no thromboembolic manifestations and no permanent pre- or postoperative heart block as the abscess forming was drained before permanent conduction damage occurs.

\section{CONCLUSION}

The unicuspid aortic valve malformation is a very rare entity with an aggressive course of valve deterioration in younger age. This deterioration is magnified in the presence of preoperative infective endocarditis, which may warrant early intervention. Surgical decision should take into consideration, the valve condition, aortic root, dimensions of adjacent aortic segments, and patient counseling for optimum results.

\section{REFERENCES}

2014 ESC Guidelines on the diagnosis and treatment of aortic diseases. 2014. European Heart Journal. 35(41):2873-2926.

Chalwade RS. 2019. Rare Case of Unicuspid Unicommissural Aortic Valve Presenting in an Adult as Infective Endocarditis, Aortic Root Abscess, and Complete Heart Block. J Indian Acad Echocardiogr Cardiovasc Imaging. 3:183-4.

Mookadam F, Thota VR, Garcia-Lopez AM, Emani UR, Alharthi MS, Zamorano J, et al. 2010. Unicuspid aortic valve in adults: a systematic review. J Heart Valve Dis. 19(1):79-85.

Mookadam F, Thota VR, Lopez AM, Emani UR, Tajik AJ. 2010. Unicuspid aortic valve in children: a systematic review spanning four decades. J Heart Valve Dis. 19(6):678-83.

Ravi D, Pir MS, Saqib N, Patel G, Abughnia H. 2019. A Rare Case of Unicuspid Aortic Valve with Postoperative Heart Block. Cureus. 11(7):e5286. Published Jul 31. 


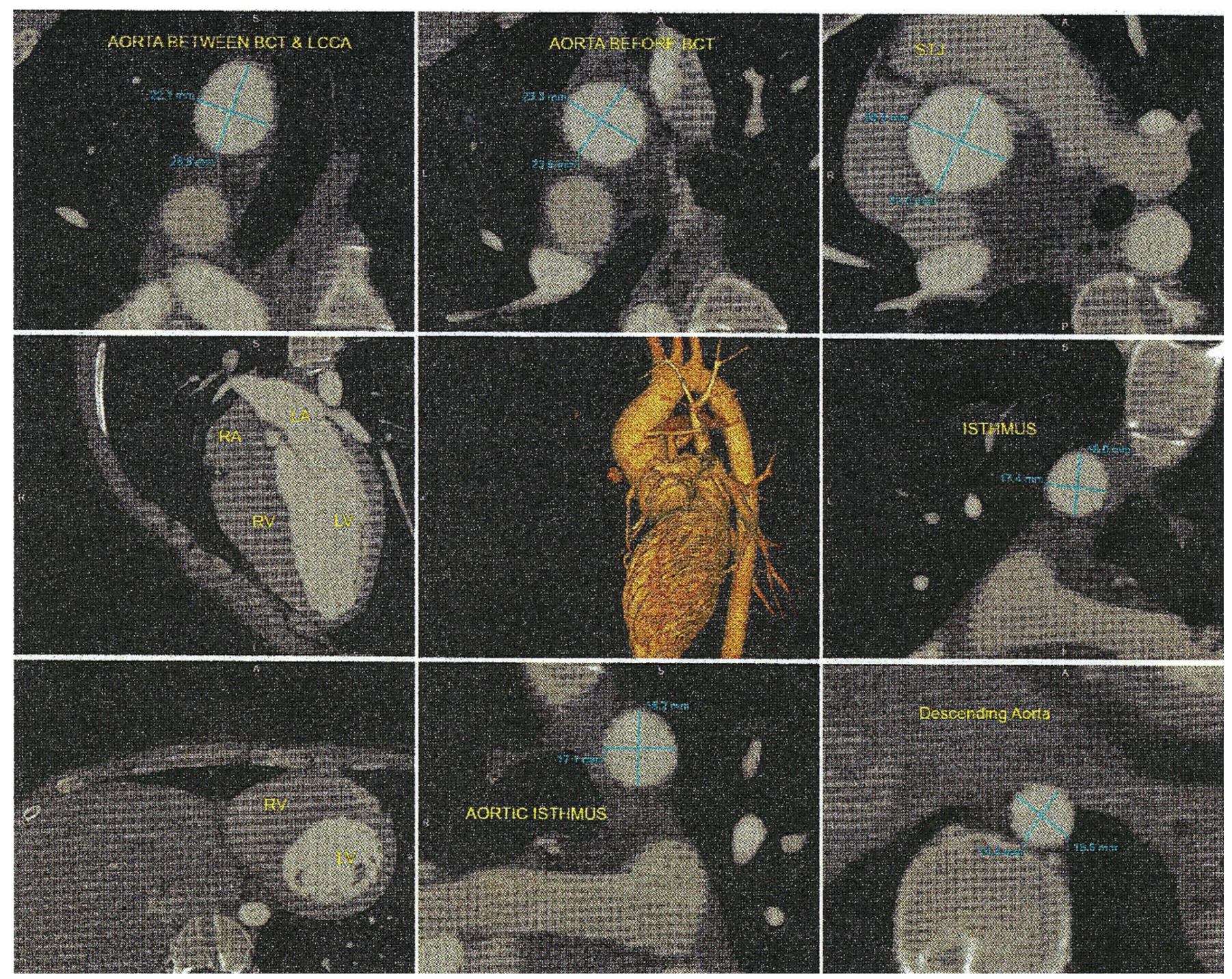

Supplementary Figure 4. MSCT showing aortic measurements at different levels.

Roberts WC, Ko JM. 2005. Frequency by decades of unicuspid, bicuspid, and tricuspid aortic valves in adults having isolated aortic valve replacement for aortic stenosis, with or without associated aortic regurgitation. Circulation. 111(7):920-5.

Singh S, Ghayal P, Mathur A, et al. 2015. Unicuspid unicommissural aortic valve: an extremely rare congenital anomaly. Tex Heart Inst J. 42(3):273-276. Published Jun 1.

Yuzawa-Tsukada N, Tanaka TD, Morimoto S, Yoshimura M. 2019. Unicuspid aortic valve concomitant with aortic insufficiency presenting with infectious endocarditis: a case report. Journal of Medical Case Reports. 13(1). 